\section{Neuer Direktor des Instituts für Technikfolgen-Abschätzung in Wien}

\author{
Von Michael Nentwich, ITA Wien
}

Der langjährige Direktor des Instituts für Technikfolgen-Abschätzung (ITA), Gunther Tichy, trat mit Ende 2005 in den wohlverdienten Ruhestand. Er hatte die von der Österreichischen Akademie der Wissenschaften vorgesehene Altersgrenze erreicht. Seine Nachfolge tritt nach einer internationalen Ausschreibung Michael Nentwich an. Dieser Beitrag blickt auf 14 Jahre ITA unter der Leitung von Tichy zurück, stellt seinen Nachfolger vor und wirft einen Blick in die Zukunft des ITA.

Erst im Juni 2005 feierte das ITA die vor zwanzig Jahren erfolgte Institutionalisierung von Technikbewertung / Technikfolgenabschätzung in Österreich an der ÖAW. Der Ökonom Gunther Tichy prägte die letzten 14 Jahre - eine Phase, die wir in der jüngst erschienen Festschrift zu seinen Ehren mit den Worten „Konsolidierung und Differenzierung" beschrieben haben ${ }^{1}$. Tichys Verankerung in der Österreichischen Akademie der Wissenschaften (ÖAW) (er wurde 1987 zum korrespondierenden und 1994 zum wirklichen Mitglied der philosophisch-historischen Klasse gewählt) und in der österreichischen Technologiepolitik waren für die institutionelle Absicherung des Instituts mitentscheidend. So wurde die ursprünglich auf zweimal drei Jahre befristete Forschungsstelle für Technikbewertung (FTB) nach einer externen Evaluierung 1994 in ein dauerhaftes Institut der ÖAW umgewandelt. Der inhaltliche Schwerpunkt der Arbeiten der FTB und später des ITA unter Tichy lag im Bereich der neuen Informations- und Kommunikationstechnologien (IKT). Unter seiner Leitung fächerte sich das Spektrum der Arbeiten weiter auf und seit Mitte der 1990er Jahre widmete sich das ITA in seinen Arbeiten umweltrelevanten Technologien, dem Umgang mit der Biotechnologie sowie Fragen der Informationsgesellschaft und des Health Technology Assessments.

Eine für den Bekanntheitsgrad des Instituts wichtige Studie war das so genannte Technologie-Delphi Austria, welches unter Feder- führung von Tichy in den Jahren 1996 bis 1998 Chancen auf technologiepolitische Themenführerschaft Österreichs in sieben Technologiefeldern analysierte. Daneben wurde auch der methodisch-institutionelle Aspekt von TA nicht vernachlässigt. Heute deckt das ITA in seinen fünf Arbeitsschwerpunkten ein breites Spektrum von Fragestellungen ab und versucht über eine starke interne Vernetzung den Herausforderungen neuer Querschnittstechnologien gerecht zu werden.

Der zum 1. Januar 2006 bestellte neue Direktor Michael Nentwich ist ursprünglich Jurist und Politikwissenschafter. Er hat an der Wirtschaftsuniversität (WU) Wien, der Universität Wien und am Europakolleg in Brügge / Belgien studiert. Er widmete sich in der ersten Phase seiner wissenschaftlichen Karriere vor allem verfassungspolitischen und verfassungsjuristischen Fragen der europäischen Integration. In der Folge konzentrierte er sich immer mehr auf die sozialwissenschaftliche Analyse von Technik und habilitierte schließlich 2004 im Fach Wissenschafts- und Technikforschung an der Universität Wien mit einer TA-Studie zu den Folgen der IKT für die Wissenschaft (Nentwich 2003). Seine beruflichen Stationen waren 1990 bis 1992 die FTB, wo er bereits unter Tichys Vorgänger, Ernest Braun, zur Umweltverträglichkeitsprüfung und zu den legistischen Folgen der Telekommunikation forschte; von 1991 bis 1996 die WU Wien, wo er als Universitätsassistent für Europarecht lehrte und forschte. Es folgten Gastaufenthalte 1994/95 an den politikwissenschaftlichen Instituten der Universitäten Warwick und Essex (Großbritannien) sowie 1998/99 am Max-Planck-Institut für Gesellschaftsforschung in Köln. Seit 1996 arbeitet er am ITA als wissenschaftlicher Mitarbeiter in verschiedenen Themenfeldern, sein inhaltlicher Schwerpunkt liegt im Bereich IKT. Aktuelle Projekte widmen sich elektronischen Publikationen und dem Urheberrecht in der Informationsgesellschaft; erarbeitet werden dabei Optionen für eine partizipative Gestaltung der österreichischen Technologiepolitik.

Das Team des ITA wird auch unter der neuen Führung seinen bewährten Weg weitergehen. Walter Peissl bleibt stellvertretender Direktor. Das aktuelle, Mitte letzten Jahres beschlossene mittelfristige Forschungsprogramm 2005 bis 2010 nimmt neue Fragestel- 
lungen und Entwicklungen auf und umfasst sieben Forschungsschwerpunkte:

- Technologien der Informationsgesellschaft: Themen sind hier etwa radikale Innovationen bei drahtgebundenen und mobilen Netzen und Peer-to-Peer-Netzen, Sensorik und Robotik, allgegenwärtige IT und der Einfluss des Einsatzes neuer Medien auf geographische und soziale Strukturen und Arbeitsweisen in der Wissenschaft.

- E-Governance: Hier geht es um die Möglichkeiten der IKT im Bereich des Staates; das Spektrum reicht von Verwaltungsrationalisierungen und Dienstleistungsinnovationen über die Behebung von Demokratiedefiziten bis zur effizienten Steuerung in politischen Mehrebenensystemen.

- Privacy: In diesem Schwerpunkt wird das Beziehungsgeflecht zwischen Technologien, Grundrechten und sozialen / politischen Folgen analysiert, um darauf aufbauend Handlungsoptionen zum Schutz der Privatsphäre abzuleiten.

- Innovative und nachhaltige Umwelttechnologien: Hier beschäftigt sich das ITA mit der Analyse von Hemmnissen bei der Verbreitung von innovativen und nachhaltigen Umwelttechnologien und der Entwicklung von Lösungen zu deren Überwindung.

- Governance von technologischem Wissen: Dieser Schwerpunkt widmet sich neuen Formen der Wissensproduktion, der Rolle von Öffentlichkeit in der Wissensproduktion, den Bearbeitungsformen von Wissensund Wertekonflikten und der Technikgestaltung nach politischen und gesellschaftlichen Zielvorgaben.

- Sicherheitsforschung: Wichtig sind hier die Themen Verunsicherung und Vertrauen, Verletzlichkeit der Informationsgesellschaft, Sicherheitstechnologien auf dem Prüfstand sowie Vorausschauende Technologieforschung.

- Monitoring: Das ITA beobachtet technologische Entwicklungen, versucht Potenziale von Emerging Technologies bzw. neuen Anwendungen zu erkennen und in einer ersten Annäherung mögliche - intendierte und nicht intendierte - Folgen (vorläufig) abzuschätzen.
Der langjährige Schwerpunkt „Health Technology Assessment" wird noch in diesem Jahr infolge einer Ausgründung in ein eigenes HTA-Institut unter Leitung von Claudia Wild auslaufen.

Fortgesetzt wird hingegen die Tradition der jährlichen internationalen TA-Konferenz in Wien. In diesem Jahr findet die Tagung am 29. Mai statt und ist dem Thema „Vermessen, codiert, entschlüsselt? Potenziale und Risken der zunehmenden Datenverfügbarkeit" gewidmet. Als Keynote-Speakers haben der Techniksoziologe Werner Rammert und der Molekularbiologe Giulio Superti-Furga zugesagt (siehe hierzu die Ankündigung in diesem Heft, oder im Internet unter http:/www.itas.fzk.de/veranstaltung/ 2006/000499.htm).

\section{Anmerkung}

1) Peissl, Nentwich 2005, S. 13; siehe dazu auch die Rezension von A. Grunwald in diesem Heft.

\section{Literatur}

Nentwich, M., 2003: Cyberscience: Research in the Age of the Internet. Vienna: Austrian Academy of Sciences Press; http://hw.oeaw.ac.at/3188-7 (letztes Abrufdatum 27.03.2006)

Peissl, W.; Nentwich, M., 2005: 20 Jahre Technikfolgenabschätzung in Österreich. In: Nentwich, M.; Peissl, W. (Hg.): Technikfolgenabschätzung in der österreichischen Praxis. Festschrift für Gunther Tichy. Wien: Verlag der der Österreichischen Akademie der Wissenschaften, S. 11-32; http://hw.oeaw.ac.at/3613-7 (letztes Abrufdatum 27.03.2006)

\section{Kontakt}

Univ.-Doz. Dr. Michael Nentwich Institut für Technikfolgen-Abschätzung Strohgasse 45/5, 1030 Wien, Österreich Tel.: +431515816583 Fax: +43171098 83

E-Mail: mnent@oeaw.ac.at Internet: http://www.oeaw.ac.at/ita 